\title{
Osso meniscal diagnosticado através de ressonância magnética: Relato de caso*
}

\section{Meniscal Ossicle Diagnosed by Magnetic Resonance Imaging: A Case Report}

\author{
Henrique Augusto Lino ${ }^{1}$ Bianca Lisa de Faria ${ }^{1}$ Ana Maria Magalhães Valle Cundari ${ }^{1}$ \\ ${ }^{1}$ Faculdade de Medicina, Universidade de Itaúna, Itaúna, Brasil \\ Endereço para Correspondência Henrique Augusto Lino, MD, \\ Rev Bras Ortop 2020;55(3):380-382. \\ Faculdade de Medicina, Universidade de Itaúna, Itaúna, MG, Brasil \\ (e-mail: henriquelino@live.com).
}

\section{Resumo \\ Palavras-chave \\ - traumatismos do joelho \\ - articulação do joelho \\ - meniscos tibiais \\ - imagem por ressonância magnética}

O osso meniscal é uma condição rara, ocasionalmente sintomática. Apesar de sua baixa incidência, é importante seu reconhecimento, uma vez que pode mimetizar outras lesões. A ressonância magnética (RM) é um método sensível e específico para seu diagnóstico. A evolução é de curso benigno e favorável ao tratamento conservador, não necessita de procedimentos diagnósticos e terapêuticos, como artroscopia, na grande maioria dos casos. No presente trabalho, foi demonstrado como o diagnóstico correto pode ser feito por RM.

Meniscal ossicle is a rare condition, which is occasionally symptomatic. Even though it has a low incidence, its diagnosis is important, because it may mimic other lesions. Magnetic resonance imaging (MRI) is a sensitive and specific method for its diagnosis. Its clinical behavior is benign and its conservative management is almost always successful, without any need for diagnostic and therapeutic procedures, such as arthroscopy. This report was able to demonstrate the correct diagnosis by MRI.

\section{Introdução}

O osso meniscal consiste em uma lesão rara, estima-se uma prevalência de $0,15 \%$ em pacientes submetidos à ressonância magnética (RM), através de um estudo seccional. ${ }^{1} \mathrm{O}$ achado pode ocorrer em exames de imagem de pacientes assintomáticos ou que foram submetidos aos estudos devido a queixas de dor articular intermitente, edema e limitação de movimento articular. ${ }^{2}$

Publicado Originalmente por Elsevier editora Ltda.

recebido

30 de Dezembro de 2017

aceito

10 de Abril de 2018
DOI https://doi.org/

10.1016/j.rbo.2018.04.004. ISSN $0102-3616$.
Apesar de debatida sua etiologia, ${ }^{3}$ o achado tem evolução benigna e, dessa forma, seu incorreto diagnóstico pode levar o paciente a procedimentos propedêuticos e terapêuticos desnecessários.

O presente relato tem por objetivo tratar de um caso, demonstrar a importância do reconhecimento de seus achados característicos à RM. ${ }^{2,4}$

\section{Relato do Caso}

Paciente masculino, branco, 35 anos, apresentava queixa de dor crônica em articulação do joelho, com piora progressiva

Copyright $\odot 2020$ by Sociedade Brasileira License terms de Ortopedia e Traumatologia. Published by Thieme Revinter Publicações Ltda, Rio de Janeiro, Brazil 


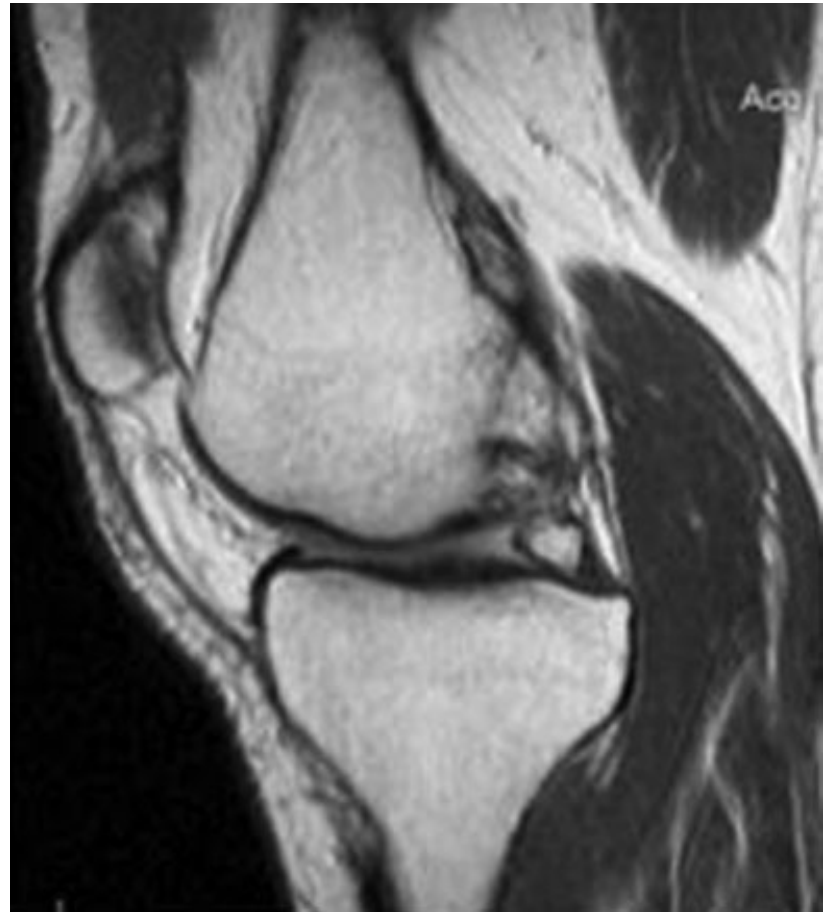

Fig. 1 Imagem ponderada em T1WI em plano sagital, demonstra estrutura triangular, com intensidade equivalente a tecido ósseo, localiza-se em topografia de corno posterior do menisco medial.

ao longo de um ano. A dor exacerbava-se aos exercícios e ao decorrer do dia e aliviava-se com analgésicos e descanso.

Era um paciente hígido, sem comorbidades. Negava história de trauma. Também não apresentava histórico familiar relevante de doenças osteomusculares, reações alérgicas ou antecedentes cirúrgicos.

Ao exame físico, demonstrou dor em compartimento medial da articulação do joelho durante a flexão ativa e passiva

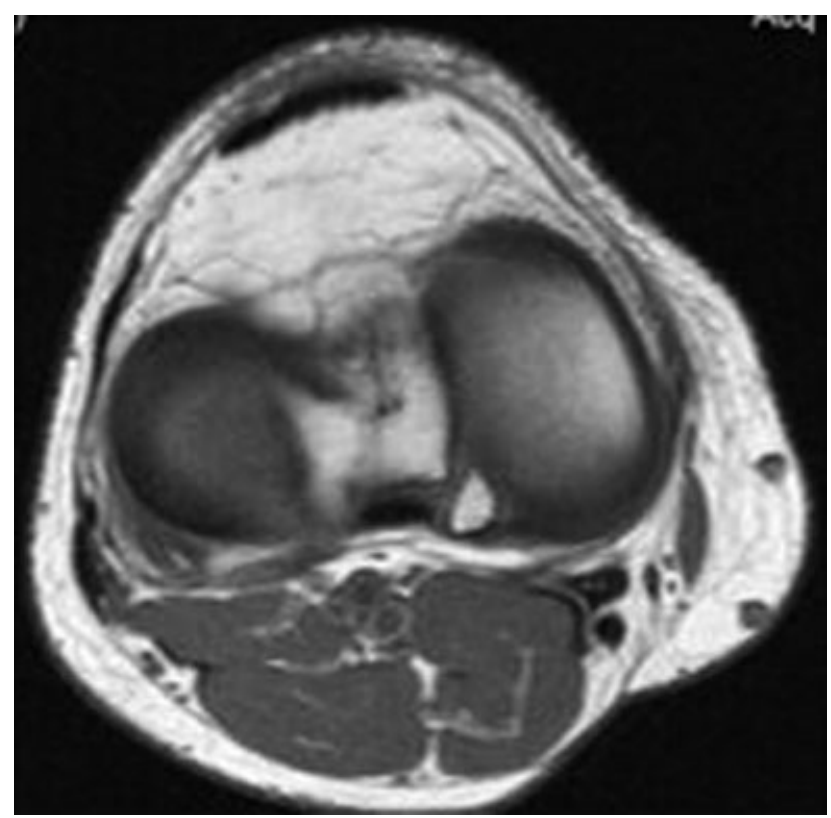

Fig. 2 Imagem ponderada em T1WI no plano axial, evidencia o osso meniscal adjacente ao corno posterior do menisco medial.

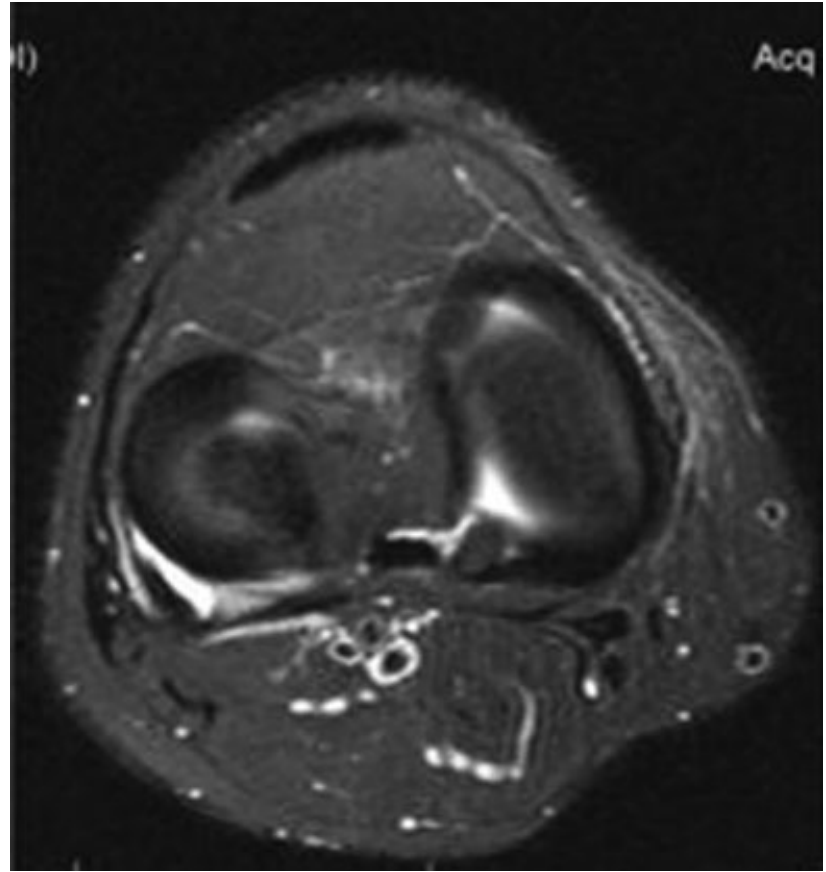

Fig. 3 Imagem ponderada em T2WI fatsat no plano axial.

da articulação. Não apresentava limitações de movimento articular, rigidez, edema, deformidades, sinais flogísticos ou outras alterações osteomusculares ou de tecidos moles. Diante da apresentação, o médico assistente optou por indicar RM para investigação de uma suspeita de ruptura meniscal.

O exame de imagem foi feito na semana subsequente, com aquisição de imagens em T1, T2 fatsat e Gradient Recoil Echo T2. As imagens evidenciaram uma estrutura de formato arredondado ou triangular, com contornos bem definidos e intensidade similar ao tecido ósseo, em todas as sequências, em topografia de corno posterior do menisco medial, como demonstrado nas - Figuras 1-4. Consequentes ao diagnóstico

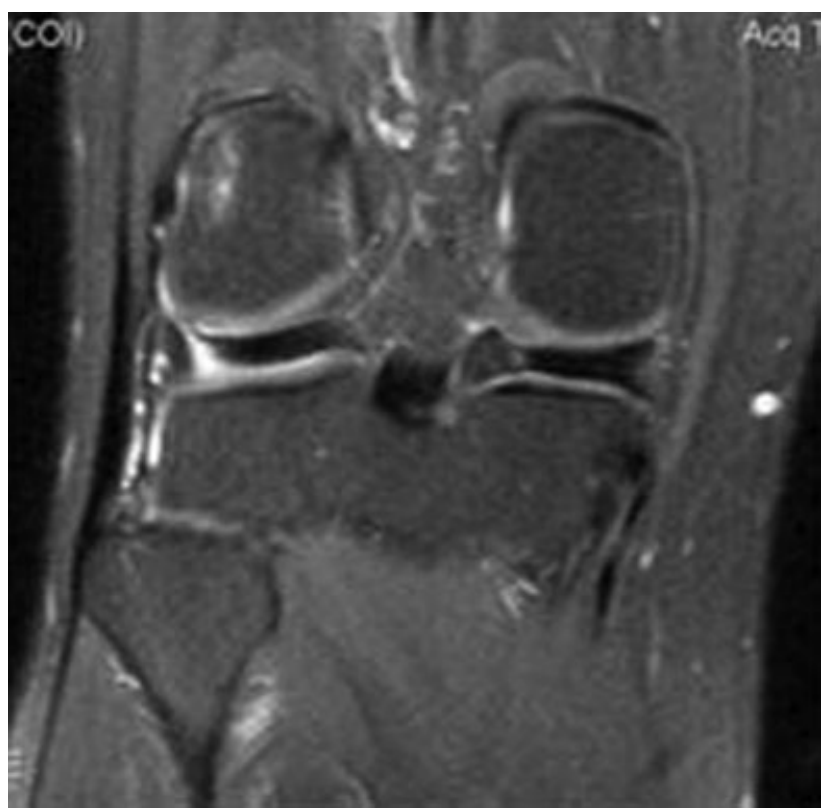

Fig. 4 Imagem coronal obtida em GRE T2. 
foram oferecidas as opções de tratamento com ressecção artroscópica do osso meniscal ou tratamento clínico. 0 paciente optou pela conduta conservadora, com uso de analgésicos e anti-inflamatórios para controle dos sintomas e acompanhamento clínico. A evolução foi favorável, sem necessidade de terapêutica adicional.

\section{Discussão}

A primeira descrição com repercussão sobre a condição foi de Burrows, $^{5}$ em 1934, com estudo clínico, radiológico e anatomopatológico do caso. Desde então, foram relatados $<50$ casos em todo o mundo. ${ }^{6}$ Estima-se que sua maior incidência ocorra entre os 12 e 76 anos, com diagnóstico em média aos 26 anos; 84\% dos casos são incidentes em homens. ${ }^{4}$

A lesão pode ser assintomática, ser encontrada acidentalmente ou apresentar artralgia, como no caso relatado, e edema. Raramente associa-se a sintomas inespecíficos ou limitação de movimento articular. ${ }^{6}$ Alguns autores apontam associação do achado com trauma articular, porém não é consensual sua natureza traumática. ${ }^{7}$ Dessa forma, sua apresentação clínica é inespecífica e não permite um diagnóstico definitivo.

O método mais sensível e específico para diagnóstico da lesão é a RM, uma vez que permite caracterizá-la como isointensa ao tecido ósseo. Sua localização mais comum é em topografia de corno posterior do menisco medial. Os casos de menisco lateral, cornos anteriores ou ossos bilaterais são apresentações consideravelmente mais raras.

Referentemente aos aspectos clínicos e de imagem, corpos livres osteocondrais, condrocalcinose, ruptura meniscal, osteocondrite dissecante e osteocrondomatose sinovial são seus principais diagnósticos diferenciais. ${ }^{8}$ A maior importância em seu diagnóstico acurado consiste em evitar propedêutica e tratamentos desnecessários, uma vez que, ao contrário das condições supracitadas, seu tratamento conservador com anti-inflamatórios tende a ser suficiente para controle clínico. ${ }^{6,9}$ Ocasionalmente, pode-se optar pelo tratamento com ressecção artroscópica. 6,7,10

O osso meniscal tem apresentação característica à RM, que é um método acurado para seu diagnóstico. Ao ser corretamente diferenciado de outros potenciais diagnósticos diferenciais, é evitada propedêutica e terapêutica invasiva, uma vez que seu tratamento conservador tende a ser eficaz e evita-se maior morbidade e gastos para o paciente ou o sistema público de saúde.

\section{Conflito de Interesses}

Os autores declaram não haver conflito de interesses.

\section{Referências}

1 Schnarkowski P, Tirman PF, Fuchigami KD, Crues JV, Butler MG, Genant HK. Meniscal ossicle: radiographic and MR imaging findings. Radiology 1995;196(01):47-50

2 Devgan A, Mukhopadhyay R, Singh A, Gogna P, Singla R, Magu NK. Ossicle in anterior cruciate ligament: a rare occurrence. Case Rep Orthop 2014;2014:616715

3 Lee JH, Wang S II, Jung YR. Meniscal ossicle.J Korean Orthop Assoc. 2016;51(02):173-7. Available at: https://synapse.koreamed.org/ DOIx.php?id=10.4055/jkoa.2016.51.2.173

4 Raustol OA, Poelstra KA, Chhabra A, Diduch DR. The meniscal ossicle revisited: etiology and an arthroscopic technique for treatment. Arthroscopy 2006;22(06):687.e1-687.e3

5 Burrows HJ. Two cases of ossification in the internal semilunar cartilage. Br J Surg 1934;21:404-410

6 Van Breuseghem I, Geusens E, Pans S, Brys P. The meniscal ossicle revisited. JBR-BTR 2003;86(05):276-277

7 Ogassawara R, Zayni R, Orhant E, Noel E, Fournier Y, Hager J-P, Chambat P, Sonnery-Cottet B. Meniscal ossicle in a professional soccer player. Orthop Traumatol Surg Res 2011;97(04):443-446

8 Yen P, Hunter J, Escobedo E. Meniscal Ossicle in the Lateral Meniscus: An Unusual Variant of a Rare Entity. Radiol Case Rep 2016;4(02):289

9 Rohilla S, Yadav RK, Singh R, Devgan A, Dhaulakhandi DB. Meniscal ossicle. J Orthop Traumatol 2009;10(03):143-145

10 Ohishi T, Suzuki D, Yamamoto K, Banno T, Shimizu Y, Ohmura A, Matsuyama Y. Meniscal pullout repair following meniscal ossicle resection: a case report. Knee 2013;20(01):52-57 